\title{
The Existence of Exponential Attractor for Discrete Ginzburg-Landau Equation
}

\author{
Guangyin Du, ${ }^{1}$ Zeqi Zhu, $^{2}$ and Caidi $\mathrm{Zhao}^{3}$ \\ ${ }^{1}$ Institute of Geotechnical Engineering, Southeast University, Nanjing, Jiangsu 210096, China \\ ${ }^{2}$ State Key Laboratory of Geomechanics and Geotechnical Engineering, Institute of Rock and Soil Mechanics, \\ Chinese Academy of Sciences, Wuhan, Hubei 430071, China \\ ${ }^{3}$ Department of Mathematics and Information Science, Wenzhou University, Wenzhou, Zhejiang 325035, China
}

Correspondence should be addressed to Caidi Zhao; zhaocaidi2013@163.com

Received 17 May 2015; Accepted 13 September 2015

Academic Editor: Chris Goodrich

Copyright (C) 2015 Guangyin Du et al. This is an open access article distributed under the Creative Commons Attribution License, which permits unrestricted use, distribution, and reproduction in any medium, provided the original work is properly cited.

This paper studies the following discrete systems of the complex Ginzburg-Landau equation: $i \dot{u}_{m}-(\alpha-i \varepsilon)\left(2 u_{m}-u_{m+1}-u_{m-1}\right)+$ $i \kappa u_{m}+\beta\left|u_{m}\right|^{2 \sigma} u_{m}=g_{m}, m \in \mathbb{Z}$. Under some conditions on the parameters $\alpha, \varepsilon, \kappa, \beta$, and $\sigma$, we prove the existence of exponential attractor for the semigroup associated with these discrete systems.

\section{Introduction}

In the study of infinite dynamical systems, attractors occupy a central position (see, e.g., Chepyzhov and Vishik [1], Hale [2], Ladyzhenskaya [3], and Temam [4]). Exponential attractors are realistic objects intermediate between the global attractors and the inertial manifolds. There are several approaches for proving the existence of exponential attractors for parabolic and hyperbolic partial differential equations (PDEs) arising from mathematical physics. For example, we can refer to [5-7] for the existence of the exponential attractors for general evolution equations in Banach spaces, to [8] for the exponential attractors for reaction diffusion equations in unbounded domains, to [9] for the exponential attractors of the nonlinear wave equations, and to [10] for the exponential attractor for the generalized 2D Ginzburg-Landau equations. Also there are some references investigating the exponential attractors for lattice dynamical systems (LDSs). We can see [11-13] for the exponential attractors for firstorder LDSs; see $[14,15]$ for the pullback exponential attractors for first- and second-order LDSs; see [16, 17] for second-order nonautonomous LDSs and discrete Zakharov equations for the uniform exponential attractors.
Lattice dynamical systems (LDSs) are currently under active investigation for their wide applications in electrical engineering [18], chemical reaction theory [19, 20], laser systems [21], and biology [22]. There are many references studying the asymptotic behavior of general LDSs. For instance, we can refer to [23-25] for the existence of global attractor, to [26-28] for the uniform attractor, to [11, 14, 15] for the exponential and pullback exponential attractor, and to $[29,30]$ for the random attractor. Also, there are some concrete applications of the above theory to the discrete PDEs. We can refer to [31-33] for discrete Klein-GordonSchrödinger equations, [34] for discrete three-component reversible Gray-Scott model, [35] for discrete coupled nonlinear Schrödinger-Boussinesq equations, [36] for discrete long-wave-short-wave resonance equations, and [37] for the discrete complex Ginzburg-Landau equation.

Lattice systems including coupled ordinary differential equations, coupled map lattices, and cellular automata are spatiotemporal systems with discretization in some variables. In some cases, lattice systems arise as the spatial discretization of partial differential equations on unbounded or bounded domains. 
This paper will study the following discrete systems (lattice systems):

$$
\begin{aligned}
& i \dot{u}_{m}-(\alpha-i \varepsilon)\left(2 u_{m}-u_{m+1}-u_{m-1}\right)+i \kappa u_{m} \\
& \quad+\beta\left|u_{m}\right|^{2 \sigma} u_{m}=g_{m}, \\
& u_{m}(0)=u_{0, m}, \quad m \in \mathbb{Z},
\end{aligned}
$$

where $i$ is the unit of imaginary numbers and $\alpha, \varepsilon, \kappa, \beta, \sigma$ are parameters. Equation (1) can be regarded as a discrete analogue of the following complex Ginzburg-Landau equation on the real line:

$$
i \dot{u}+(\alpha-i \varepsilon) u_{x x}+i \kappa u+\beta|u|^{2 \sigma} u=g, \quad x \in \mathbb{R} .
$$

The complex Ginzburg-Landau equation is a simplified mathematical model for various pattern formation systems in mechanics, physics, and chemistry. We can refer to [10, 38, 39] for the detailed significations of the complex GinzburgLandau equation.

The existence of the exponential attractors for continuous complex Ginzburg-Landau equation in two-dimensional space was proved in [10]. Later, under some conditions on $\alpha, \varepsilon, \kappa, \beta, \sigma$, and $g_{m}$, [37] established the existence of global attractor for the semigroup associated with discrete systems (1)-(2). The aim of this paper is to prove the existence of exponential attractors for discrete systems (1)-(2). To this end, we will establish the following three items:

(I) The solution operators associated with (1)-(2) generate a continuous semigroup $\{S(t)\}_{t \geqslant 0}$ in the phase space $\ell^{2}$ and $\{S(t)\}_{t \geqslant 0}$ possesses a bounded and closed positively invariant set $\mathscr{B} \subset \ell^{2}$. Moreover, for any $T>0$, the map $S(t)$ is Lipschitz continuous from $[0, T] \times \mathscr{B}$ into $\mathscr{B}$.

(II) There exists a time $T_{*}$ such that the map $S\left(T_{*}\right):=S_{*}$ : $\mathscr{B} \mapsto \mathscr{B}$ is an $\alpha$-contraction on $\mathscr{B}$.

(III) The map $S_{*}$ satisfies the discrete squeezing property on $\mathscr{B}$.

Compared with previous works such as [9], here we no longer require the compactness of the invariant set $\mathscr{B}$ (this fact was first noted by Babin and Nicolaenko [8] and then by Eden et al. [6]), which can usually be obtained by the compact embedding between Sobolev spaces when studying PDEs. Note that the compact embedding theorem of Sobolev spaces seems difficult to be applicable when studying LDSs. This is caused by the discrete characteristics of LDSs which restrict us to choose the phase spaces. Fortunately, the intrinsic characteristics of LDSs enable us to use the $\alpha$-contraction property to compensate the compactness of the invariant set.

\section{Positively Invariant Set and Lipschitz Continuity}

Set

$$
\ell^{2}=\left\{u=\left(u_{m}\right)_{m \in \mathbb{Z}}, u_{m} \in \mathbb{C}: \sum_{m \in \mathbb{Z}}\left|u_{m}\right|^{2}<+\infty\right\}
$$

and equip it with the inner product and norm as

$$
\begin{aligned}
(u, v) & =\sum_{m \in \mathbb{Z}} u_{m} \bar{v}_{m}, \\
\|u\|^{2} & =(u, u), \\
u & =\left(u_{m}\right)_{m \in \mathbb{Z}}, \\
v & =\left(v_{m}\right)_{m \in \mathbb{Z}} \in \ell^{2},
\end{aligned}
$$

where $\bar{v}_{m}$ denotes the conjugate of $v_{m}$. Then $\left(\ell^{2},\|\cdot\|,(\cdot, \cdot)\right)$ is a separable Hilbert space. We now introduce the operators $A$, $B$, and $B^{*}$ on $\ell^{2}$ as follows:

$$
\begin{aligned}
(A u)_{m} & =2 u_{m}-u_{m+1}-u_{m-1}, \quad \forall m \in \mathbb{Z}, \\
(B u)_{m} & =u_{m+1}-u_{m} \\
\left(B^{*} u\right)_{m} & =u_{m-1}-u_{m}
\end{aligned}
$$

$\forall m \in \mathbb{Z}$

In fact, $B^{*}$ is the adjoint operator of $B$ and one can easily check that

$$
\begin{aligned}
& (A u, v)=\left(B^{*} B u, v\right)=(B u, B v), \\
& (B u, v)=\left(u, B^{*} v\right),
\end{aligned}
$$$$
\forall u, v \in \ell^{2}
$$

$$
\begin{gathered}
\|A u\|^{2} \leqslant 16\|u\|^{2}, \\
\|B u\|^{2} \leqslant 4\|u\|^{2}, \\
\left\|B^{*} u\right\|^{2} \leqslant 4\|u\|^{2},
\end{gathered}
$$

$$
\forall u \in \ell^{2}
$$

Using the notations introduced above, we can write problem (1)-(2) as

$$
\begin{aligned}
i \dot{u}-(\alpha-i \varepsilon) A u+i \kappa u+\beta|u|^{2 \sigma} u & =g, \\
u(0) & =u_{0},
\end{aligned}
$$

where $u=\left(u_{m}\right)_{m \in \mathbb{Z}},|u|^{2 \sigma} u=\left(\left|u_{m}\right|^{2 \sigma} u_{m}\right)_{m \in \mathbb{Z}}, g=\left(g_{m}\right)_{m \in \mathbb{Z}}$, and $u_{0}=\left(u_{m}(0)\right)_{m \in \mathbb{Z}}$.

For the well-posedness of problem (1)-(2), we have the following.

Lemma 1 (see [37]). Let $\alpha, \varepsilon, \kappa, \beta, \sigma>0$ and $g=\left(g_{m}\right)_{m \in \mathbb{Z}} \in$ $\ell^{2}$

(i) For any $u_{0} \in \ell^{2}$, problem (8)-(9) has a unique solution $u \in \mathscr{C}^{1}\left(\left[0, T_{0}\right) ; \ell^{2}\right)$ for some $T_{0}>0$. Moreover, if $T_{0}<$ $+\infty$, then $\lim _{t \rightarrow+T_{0}^{-}}\|u(t)\|=+\infty$.

(ii) For any $u_{0} \in \ell^{2}$, the solution of problem (8)-(9) satisfies

$$
\|u(t)\|^{2} \leqslant\left\|u_{0}\right\|^{2} e^{-\kappa t}+\frac{\|g\|^{2}}{\kappa^{2}}, \quad \forall t \geqslant 0
$$


Lemma 1(i) shows that, for each initial value $u_{0} \in \ell^{2}$, problem (8)-(9) possesses a unique solution. Letting $t \rightarrow$ $+\infty$, we see from (10) that, for any $u_{0} \in \ell^{2}$, the corresponding solution $u(t) \in \ell^{2}$ of problem (8)-(9) is uniformly (with respect to $t$ ) bounded for all $t \in[0,+\infty)$. Again, by Lemma 1(i), the solution exists globally; that is, problem (8)(9) is globally well-posed. The above analysis implies that the solution operators

$$
S(t): \ell^{2} \ni u_{0} \longmapsto S(t) u_{0}=u(t) \in \ell^{2}
$$

generate a continuous semigroup $\{S(t)\}_{t \geqslant 0}$ on $\ell^{2}$. We next investigate the existence of the bounded and closed positively invariant set, as well as the Lipschitz property for the semigroup $\{S(t)\}_{t \geqslant 0}$.

Lemma 2. Let $\alpha, \varepsilon, \kappa, \beta, \sigma>0$ and $g=\left(g_{m}\right)_{m \in \mathbb{Z}} \in \ell^{2}$. Then the semigroup $\{S(t)\}_{t \geqslant 0}$ possesses a bounded and closed positively invariant set $\mathscr{B} \subset \ell^{2}$.

Proof. By (10) we see that the set

$$
\mathscr{B}:=\left\{u \in \ell^{2}:\|u\| \leqslant \frac{\sqrt{2}\|g\|}{\kappa}\right\}
$$

is a bounded and closed absorbing set for $\{S(t)\}_{t \geqslant 0}$. Thus, there is a time $t_{*}:=t_{*}(\mathscr{B})$ such that $S(t) \mathscr{B} \subseteq \mathscr{B}$ for any $t \geqslant t_{*}$. Set

$$
\mathscr{B}:=\overline{\bigcup_{\tau \geqslant t_{*}} S(\tau) \mathscr{B}} .
$$

Then $\mathscr{B}$ is the bounded and closed positively invariant set for $\{S(t)\}_{t \geqslant 0}$. The proof is complete.

The positively invariant property of $\mathscr{B}$ implies that

$$
S(t) \mathscr{B} \subseteq \mathscr{B} \subseteq \mathscr{B}, \quad \forall t \geqslant 0 .
$$

Lemma 3. Let $\alpha, \varepsilon, \kappa, \delta>0, \sigma \geqslant 1 / 2$, and $g=\left(g_{m}\right)_{m \in \mathbb{Z}} \in$ $\ell^{2}$. Then the semigroup $\{S(t)\}_{t \geqslant 0}$ is Lipschitz continuous from $[0, T] \times \mathscr{B}$ into $\mathscr{B}$ for each $\mathrm{T}>0$.

Proof. Let $u_{0}, v_{0} \in \mathscr{B}, S(t) u_{0}=u(t)=\left(u_{m}(t)\right)_{m \in \mathbb{Z}}, S(t) v_{0}=$ $v(t)=\left(v_{m}(t)\right)_{m \in \mathbb{Z}}$, and $w(t)=u(t)-v(t)$. By $(8)$,

$$
\begin{aligned}
& i \dot{w}-(\alpha-i \varepsilon) A w+i \kappa w+\beta|u|^{2 \sigma} u-\beta|v|^{2 \sigma} v=0, \\
& w(0)=u_{0}-v_{0} .
\end{aligned}
$$

Using $i w(t)$ to take inner product $(\cdot, \cdot)$ with both sides of (15) and then taking the real part, we obtain

$$
\begin{aligned}
& \frac{1}{2} \frac{\mathrm{d}}{\mathrm{d} t}\|w(t)\|^{2}+\varepsilon\|B u\|^{2}+\kappa\|w(t)\|^{2} \\
& \quad-\operatorname{Im} \beta \sum_{m \in \mathbb{Z}}\left(\left|u_{m}\right|^{2 \sigma} u_{m}-\left|v_{m}\right|^{2 \sigma} v_{m}\right) \bar{w}_{m}=0 .
\end{aligned}
$$

Now set $f(x)=x^{2 \sigma}, x \in \mathbb{R}_{+}$. Since $\sigma \geqslant 1 / 2, f^{\prime}(x)=2 \sigma x^{2 \sigma-1}$ is continuous and increasing on $\mathbb{R}_{+}$. By Cauchy inequality,

$$
\begin{aligned}
- & \operatorname{Im} \beta \sum_{m \in \mathbb{Z}}\left(\left|u_{m}\right|^{2 \sigma} u_{m}-\left|v_{m}\right|^{2 \sigma} v_{m}\right) \bar{w}_{m} \\
\leqslant & \frac{\kappa}{2}\|w(t)\|^{2} \\
& +\frac{\beta^{2}}{2 \kappa} \sum_{m \in \mathbb{Z}}\left(f\left(\left|u_{m}\right|\right) u_{m}-f\left(\left|v_{m}\right|\right) v_{m}\right)^{2} .
\end{aligned}
$$

Using mean value theorem, (12), and (14), we get

$$
\begin{gathered}
\sum_{m \in \mathbb{Z}}\left(f\left(\left|u_{m}\right|\right) u_{m}-f\left(\left|v_{m}\right|\right) v_{m}\right)^{2} \\
\leqslant\left(2+8 \sigma^{2}\right)\left(\frac{2\|g\|^{2}}{\kappa^{2}}\right)^{2 \sigma}\|w\|^{2} .
\end{gathered}
$$

It then follows from (17)-(19) that

$$
\frac{\mathrm{d}}{\mathrm{d} t}\|w(t)\|^{2}+(\kappa-\delta)\|w(t)\|^{2} \leqslant 0,
$$

where

$$
\delta=\delta(\kappa, \beta, \sigma,\|g\|):=\frac{2 \beta^{2}}{\kappa} \cdot\left(1+4 \sigma^{2}\right)\left(\frac{2\|g\|^{2}}{\kappa^{2}}\right)^{2 \sigma} .
$$

Applying Gronwall inequality to (20) yields

$$
\begin{array}{r}
\|w(t)\|^{2}=\left\|S(t) u_{0}-S(t) v_{0}\right\|^{2} \leqslant\|w(0)\|^{2} e^{(\delta-\kappa) t}, \\
\forall t \geqslant 0,
\end{array}
$$

and, for any $T>0$,

$$
\left\|S(t) u_{0}-S(t) v_{0}\right\| \leqslant\left\|u_{0}-v_{0}\right\| e^{(\delta-\kappa) T / 2}, \quad, \quad \forall t \in[0, T] .
$$

The proof is complete.

\section{Existence of Exponential Attractor}

For each positive number $M$, we define the orthogonal projection $P_{M}: \ell^{2} \mapsto \ell^{2}$ as

$$
\left(P_{M} u\right)_{m}= \begin{cases}u_{m}, & |m| \leqslant M ; \\ 0, & |m|>M\end{cases}
$$

and set $Q_{M}=I-P_{M}$, where $I$ is the identity operator on $\ell^{2}$.

We next make some assumptions on the numbers $\alpha, \kappa, \beta, \sigma$, and $g_{m}$ :

(H) Assume $g=\left(g_{m}\right)_{m \in \mathbb{Z}} \in \ell^{2}, \alpha, \kappa, \beta$ are positive, $\sigma \geqslant 1 / 2$, and

$$
\kappa>\delta
$$

where $\delta$ is defined by (21).

The definitions of $\alpha$-contraction and discrete squeezing property can be found in $[2,6]$. 
Lemma 4. Let assumption $(\mathbf{H})$ hold. Then there exists a time $T_{*}$ such that the operator $S\left(T_{*}\right):=S_{*}: \mathscr{B} \mapsto \mathscr{B}$ is an $\alpha$ contraction on $\mathscr{B}$.

Proof. Let $u_{0}, v_{0} \in \mathscr{B}, S(t) u_{0}=u(t)=\left(u_{m}(t)\right)_{m \in \mathbb{Z}}, S(t) v_{0}=$ $u(t)=\left(v_{m}(t)\right)_{m \in \mathbb{Z}}$, and $w(t)=u(t)-v(t)$. By $(20)$, we have for any $M \in \mathbb{N}$ that

$$
\begin{gathered}
\frac{\mathrm{d}}{\mathrm{d} t}\|w(t)\|^{2}+\kappa\|w(t)\|^{2} \leqslant \delta\|w(t)\|^{2} \\
=\delta\left(\left\|P_{M} w(t)\right\|^{2}+\left\|Q_{M} w(t)\right\|^{2}\right),
\end{gathered}
$$

which, together with (25), gives

$$
\frac{\mathrm{d}}{\mathrm{d} t}\|w(t)\|^{2}+(\kappa-\delta)\|w(t)\|^{2} \leqslant \delta\left\|P_{M} w(t)\right\|^{2},
$$

Thus we have

$$
t \geqslant t_{*} \text {. }
$$

$$
\frac{\mathrm{d}}{\mathrm{d} t}\left(e^{(\kappa-\delta) t}\|w(t)\|^{2}\right) \leqslant \delta e^{(\kappa-\delta) t}\left\|P_{M} w(t)\right\|^{2}, \quad t \geqslant t_{*} .
$$

Integrating both sides of (28) over $\left[t_{*}, T\right]$ with $T>t_{*}$ and then using (22), we obtain

$$
\begin{aligned}
\|w(T)\|^{2} \leqslant & e^{-(\kappa-\delta) T}\|w(0)\|^{2} \\
& +\frac{\delta}{\kappa-\delta} \max _{s \in\left[t_{*}, T\right]}\left\|P_{M} w(s)\right\|^{2}, \quad T \geqslant t_{*} .
\end{aligned}
$$

Now we choose

$$
\begin{gathered}
T_{*}=\max \left\{t_{*}+\frac{\ln 256}{\kappa-\delta}+\frac{(\kappa+\delta) t_{*}+\ln 256}{2 \kappa},\right. \\
\left.\frac{\ln ((\kappa+\delta) / 2048 \beta \sigma)(\kappa / 2\|g\|)^{2 \sigma}}{\kappa-\delta}\right\}
\end{gathered}
$$

and it follows from (29) that

$$
\left\|w\left(T_{*}\right)\right\|^{2} \leqslant \frac{\|w(0)\|^{2}}{256}+\frac{\delta}{\kappa-\delta} \max _{s \in\left[t_{*}, T_{*}\right]}\left\|P_{M} w(s)\right\|^{2} .
$$

Proceeding as that as [11] did, we can show $\sqrt{\delta /(\kappa-\delta)} \max _{s \in\left[t_{*}, T_{*}\right]}\left\|P_{M} w(s)\right\|$ is a precompact pseudometric on $\mathscr{B}$, which, together with (31) and [2, Lemma 2.3.6], gives the desired result.

Remark 5. Since Lemma 4 holds for any $M \in \mathbb{N}$, we can specify some $M_{*}$ (see (40)). Then $T_{*}$ is chosen such that both $e^{(\delta-\kappa) T_{*}} \leqslant 1 / 256$ and (41) hold.

Lemma 6. Let assumption $(\mathbf{H})$ hold. Then the operator $S_{*}$ : $\mathscr{B} \mapsto \mathscr{B}$ satisfies the discrete squeezing property on $\mathscr{B}$.

Proof. Define a smooth function $\chi(x) \in \mathscr{C}\left(\mathbb{R}_{+},[0,1]\right)$ (see, e.g., [33]) such that

$$
\begin{gathered}
\chi(x)= \begin{cases}0, & 0 \leqslant x \leqslant 1 \\
1, & x \geqslant 2,\end{cases} \\
\left|\chi^{\prime}(x)\right| \leqslant \chi_{0} \quad \text { (constant) }, \forall x \in \mathbb{R}_{+} .
\end{gathered}
$$

Let $u_{0}, v_{0} \in \mathscr{B}, S(t) u_{0}=u(t)=\left(u_{m}(t)\right)_{m \in \mathbb{Z}}, S(t) v_{0}=v(t)=$ $\left(v_{m}(t)\right)_{m \in \mathbb{Z}}$, and $w(t)=u(t)-v(t)$. Set $y_{m}=\chi\left(|m| / M_{*}\right) w_{m}$ for each $m \in \mathbb{Z}$ and $y=\left(y_{m}\right)_{m \in \mathbb{Z}}$, where $M_{*}$ is a positive integer that will be specified later. Using $i y(t)$ to take inner product $(\cdot, \cdot)$ with both sides of (15) and then taking the real part, we obtain

$$
\begin{aligned}
& \frac{1}{2} \frac{\mathrm{d}}{\mathrm{d} t}\left(\sum_{m \in \mathbb{Z}} \chi\left(\frac{|m|}{M_{*}}\right)\left|w_{m}\right|^{2}\right) \\
& \quad+\operatorname{Re}(i \alpha+\varepsilon) \sum_{m \in \mathbb{Z}}(B w)_{m}(B \bar{y})_{m} \\
& \quad-\operatorname{Im} \beta \sum_{m \in \mathbb{Z}} \chi\left(\frac{|m|}{M_{*}}\right)\left(\left|u_{m}\right|^{2 \sigma} u_{m}-\left|v_{m}\right|^{2 \sigma} v_{m}\right) \bar{w}_{m} \\
& \quad+\kappa \sum_{m \in \mathbb{Z}} \chi\left(\frac{|m|}{M_{*}}\right)\left|w_{m}\right|^{2}=0 .
\end{aligned}
$$

By (31) and $[37,(4.8)]$, we have for any $t \geqslant t_{*}$ that

$$
\begin{aligned}
& \operatorname{Re}(i \alpha+\varepsilon) \sum_{m \in \mathbb{Z}}(B w(t))_{m}(B \bar{y}(t))_{m} \geqslant-\frac{2 \alpha \chi_{0}}{M_{*}}\|w\|^{2}, \\
& -\operatorname{Im} \beta \sum_{m \in \mathbb{Z}} \chi\left(\frac{|m|}{M_{*}}\right)\left(f\left(\left|u_{m}\right|\right) u_{m}-f\left(\left|v_{m}\right|\right) v_{m}\right) \bar{w}_{m} \\
& \geqslant-2 \beta \sigma\left(\frac{2\|g\|}{\kappa}\right)^{2 \sigma}\|w\|^{2} .
\end{aligned}
$$

Taking (33)-(34) into account, we obtain

$$
\begin{aligned}
& \frac{\mathrm{d}}{\mathrm{d} t}\left(\sum_{m \in \mathbb{Z}} \chi\left(\frac{|m|}{M_{*}}\right)\left|w_{m}\right|^{2}\right)+2 \kappa \sum_{m \in \mathbb{Z}} \chi\left(\frac{|m|}{M_{*}}\right)\left|w_{m}\right|^{2} \\
& \quad \leqslant C\|w\|^{2}, \quad \forall t \geqslant t_{*},
\end{aligned}
$$

where $C:=4 \chi_{0} \alpha / M_{*}+4 \beta \sigma(2\|g\| / \kappa)^{2 \sigma}$. By (22) and (35), we have for any $t \geqslant t_{*}$ that

$$
\begin{aligned}
& \frac{\mathrm{d}}{\mathrm{d} t}\left(\sum_{m \in \mathbb{Z}} \chi\left(\frac{|m|}{M_{*}}\right)\left|w_{m}\right|^{2}\right)+2 \kappa \sum_{m \in \mathbb{Z}} \chi\left(\frac{|m|}{M_{*}}\right)\left|w_{m}\right|^{2} \\
& \quad \leqslant C e^{(\delta-\kappa) t}\|w(0)\|^{2}, \\
& \frac{\mathrm{d}}{\mathrm{d} t}\left(\sum_{m \in \mathbb{Z}} \chi\left(\frac{|m|}{M_{*}}\right) e^{2 \kappa t}\left|w_{m}\right|^{2}\right) \leqslant C e^{(\delta+\kappa) t}\|w(0)\|^{2} .
\end{aligned}
$$

Integrating both sides of (37) over $\left[t_{*}, T\right]$ with $T \geqslant t_{*}$, we then get

$$
\begin{aligned}
& \sum_{m \in \mathbb{Z}} \chi\left(\frac{|m|}{M_{*}}\right) e^{2 \kappa T}\left|w_{m}(T)\right|^{2} \\
& \leqslant e^{2 \kappa t_{*}}\left\|w\left(t_{*}\right)\right\|^{2}+\frac{C}{\kappa+\delta} e^{(\delta+\kappa) T}\|w(0)\|^{2} .
\end{aligned}
$$


Again from (22), we obtain that

$$
\begin{aligned}
& \left\|Q_{M_{*}} w(T)\right\|^{2} \\
& \quad \leqslant\left(e^{(\delta+\kappa) t_{*}-2 \kappa T}+\frac{C}{\kappa+\delta} e^{(\delta-\kappa) T}\right)\|w(0)\|^{2},
\end{aligned}
$$

$$
T \geqslant t_{*} .
$$

We now take

$$
M_{*}=\frac{2048 \chi_{0} \alpha}{\kappa+\delta}
$$

and then from (25), (30), and (39), we have

$$
\begin{aligned}
e^{(\delta+\kappa) t_{*}-2 \kappa T_{*}} & \leqslant \frac{1}{256}, \\
\frac{4 \chi_{0} \alpha}{(\kappa+\delta) M_{*}} e^{(\delta-\kappa) T_{*}} & \leqslant \frac{1}{512}, \\
\frac{4 \sigma \beta}{\kappa+\delta}\left(\frac{2\|g\|}{\kappa}\right)^{2 \sigma} e^{(\delta-\kappa) T_{*}} & \leqslant \frac{1}{512} .
\end{aligned}
$$

Thus $\left\|Q_{2 M_{*}} w\left(T_{*}\right)\right\|^{2}=\left\|Q_{2 M_{*}}\left(S_{*} u_{0}-S_{*} v_{0}\right)\right\|^{2} \leqslant$ $(1 / 128)\|w(0)\|^{2}=(1 / 128)\left\|u_{0}-v_{0}\right\|^{2}$. Therefore, we can claim that if $\left\|P_{2 M_{*}}\left(S_{*} u_{0}-S_{*} v_{0}\right)\right\| \leqslant\left\|Q_{2 M_{*}}\left(S_{*} u_{0}-S_{*} v_{0}\right)\right\|$, then

$$
\begin{aligned}
\left\|S_{*} u_{0}-S_{*} v_{0}\right\|^{2}= & \sum_{|m| \leq 2 M_{*}}\left|w_{m}\left(T_{*}\right)\right|^{2} \\
& +\sum_{|m| \geq 2 M_{*}}\left|w_{m}\left(T_{*}\right)\right|^{2} \\
= & \left\|P_{2 M_{*}}\left(S_{*} u_{0}-S_{*} v_{0}\right)\right\|^{2} \\
& +\left\|Q_{2 M_{*}}\left(S_{*} u_{0}-S_{*} v_{0}\right)\right\|^{2} \\
\leqslant & 2\left\|Q_{2 M_{*}}\left(S_{*} u_{0}-S_{*} v_{0}\right)\right\|^{2} \\
\leqslant & \frac{1}{64}\left\|u_{0}-v_{0}\right\|^{2} .
\end{aligned}
$$

The proof is complete.

Taking Lemmas 2, 3, 4, and 6 and [7, Theorem 3.1] into account, we now can state the main result of this paper as follows.

Theorem 7. Let assumption (H) hold. Then, one has the following:

(1) $S_{*}$ has an exponential attractor $\mathscr{A}_{*}$ on $\mathscr{B}$ which satisfies the following:

(i) $\mathscr{M} \subseteq \mathscr{A}_{*} \subseteq \mathscr{B}$, where $\mathscr{M}$ is the global attractor of $\{S(t)\}_{t \geqslant 0}$

(ii) $S_{*} \mathscr{A}_{*} \subseteq \mathscr{A}_{*}$; that is, $\mathscr{A}_{*}$ is positively invariant under $S_{*}$; (iii) $\mathscr{A}_{*}$ has finite fractal dimension $\operatorname{Dim}_{f}\left(\mathscr{A}_{*}\right)$;

(iv) there exist two constants $c_{1}$ and $c_{2}$ such that, for each $u \in \mathscr{B}$ and every positive integer $k$, $\operatorname{Dist}\left(S_{*}^{k} u, \mathscr{A}\right) \leqslant c_{1} e^{-c_{2} k}$;

(2) $\mathscr{A}=\bigcup_{0 \leqslant t \leqslant T_{*}} S(t) \mathscr{A}_{*}$ is an exponential attractor for $\{S(t)\}_{t \geqslant 0}$ on $\mathscr{B}$ and $\operatorname{Dim}_{f}(\mathscr{A}) \leqslant \operatorname{Dim}_{f}\left(\mathscr{A}_{*}\right)+1$.

\section{Conflict of Interests}

The authors declare that there is no conflict of interests regarding the publication of this paper.

\section{Acknowledgment}

This work is supported by NSFC with Grants nos. 41372264, 51279202, 41372308, and 91215301.

\section{References}

[1] V. V. Chepyzhov and M. I. Vishik, Attractors for Equations of Mathematical Physics, vol. 49, American Mathematical Society, Providence, RI, USA, 2002.

[2] J. K. Hale, Asymptotic Behavior of Dissipative Systems, American Mathematical Society, Providence, RI, USA, 1988.

[3] O. Ladyzhenskaya, Attractors for Semigroups and Evolution Equations, Cambridge University Press, Cambridge, UK, 1991.

[4] R. Temam, Infinite Dimensional Dynamical Systems in Mechanics and Physics, Springer, Berlin, Germany, 2nd edition, 1997.

[5] L. Dung and B. Nicolaenko, "Exponential attractors in Banach spaces," Journal of Dynamics and Differential Equations, vol. 13, no. 4, pp. 791-806, 2001.

[6] A. Eden, C. Foias, and V. Kalantarov, "A remark on two constructions of exponential attractors for $\alpha$-contractions," Journal of Dynamics and Differential Equations, vol. 10, no. 1, pp. 37-45, 1998.

[7] A. Eden, C. Foias, B. Nicolaenko, and R. Temam, Exponential Attractors for Dissipative Evolution Equation, vol. 37 of WileyMasson Series Research in Applied Mathematics, John Wiley \& Sons, Paris, France, 1994.

[8] A. V. Babin and B. Nicolaenko, "Exponential attractors of reaction-diffusion systems in an unbounded domain," Journal of Dynamics and Differential Equations, vol. 7, no. 4, pp. 567590, 1995.

[9] Z. Dai and D. Ma, "Exponential attractors of the nonlinear wave equations," Chinese Science Bulletin, vol. 43, no. 16, pp. 1331-1335, 1998.

[10] C. F. Liu and Z. D. Dai, "Exponential attractors for the generalized 2D Ginzburg-Landau equations in Banach spaces," Acta Mathematicae Applicatae Sinica, vol. 28, no. 1, pp. 134-142, 2005.

[11] A. Y. Abdallah, "Exponential attractors for first-order lattice dynamical systems," Journal of Mathematical Analysis and Applications, vol. 339, no. 1, pp. 217-224, 2008.

[12] X. Fan, "Exponential attractor for a first-order dissipative lattice dynamical system," Journal of Applied Mathematics, vol. 2008, Article ID 354652, 8 pages, 2008.

[13] X. Han, "Exponential attractors for lattice dynamical systems in weighted spaces," Discrete and Continuous Dynamical Systems. Series A, vol. 31, no. 2, pp. 445-467, 2011. 
[14] S. Zhou and X. Han, "Pullback exponential attractors for non-autonomous lattice systems," Journal of Dynamics and Differential Equations, vol. 24, no. 3, pp. 601-631, 2012.

[15] S. Zhou, H. Chen, and Z. Wang, "Pullback exponential attractor for second order nonautonomous lattice system," Discrete Dynamics in Nature and Society, vol. 2014, Article ID 237027, 10 pages, 2014.

[16] A. Y. Abdallah, "Exponential attractors for second order lattice dynamical systems," Communications on Pure and Applied Analysis, vol. 8, no. 3, pp. 803-813, 2009.

[17] S. Zhou and X. Han, "Uniform exponential attractors for nonautonomous KGS and Zakharov lattice systems with quasiperiodic external forces," Nonlinear Analysis: Theory, Methods \& Applications, vol. 78, pp. 141-155, 2013.

[18] S. N. Chow and J. M. Paret, "Pattern formation and spatial chaos in lattice dynamical systems. I," IEEE Transactions on Circuits Systems, vol. 42, no. 10, pp. 746-751, 1995.

[19] T. Erneux and G. Nicolis, "Propagating waves in discrete bistable reaction-diffusion systems," Physica D. Nonlinear Phenomena, vol. 67, no. 1-3, pp. 237-244, 1993.

[20] R. Kapral, "Discrete models for chemically reacting systems," Journal of Mathematical Chemistry, vol. 6, no. 2, pp. 113-163, 1991.

[21] L. Fabiny, P. Colet, R. Roy, and D. Lenstra, "Coherence and phase dynamics of spatially coupled solid-state lasers," Physical Review A, vol. 47, no. 5, pp. 4287-4296, 1993.

[22] J. P. Keener, "Propagation and its failure in coupled systems of discrete excitable cells," SIAM Journal on Applied Mathematics, vol. 47, no. 3, pp. 556-572, 1987.

[23] P. W. Bates, K. Lu, and B. Wang, "Attractors for lattice dynamical systems," International Journal of Bifurcation and Chaos in Applied Sciences and Engineering, vol. 11, no. 1, pp. 143-153, 2001.

[24] S.-N. Chow, "Lattice dynamical systems," in Dynamical Systems, vol. 1822 of Lecture Notes in Mathematics, pp. 1-102, Springer, Berlin, Germany, 2003.

[25] S. Zhou and W. Shi, "Attractors and dimension of dissipative lattice systems," Journal of Differential Equations, vol. 224, no. 1, pp. 172-204, 2006.

[26] S. Zhou, C. Zhao, and Y. Wang, "Finite dimensionality and upper semicontinuity of compact kernel sections of nonautonomous lattice systems," Discrete and Continuous Dynamical Systems A, vol. 21, no. 4, pp. 1259-1277, 2008.

[27] C. Zhao and S. Zhou, "Attractors of retarded first order lattice systems," Nonlinearity, vol. 20, no. 8, pp. 1987-2006, 2007.

[28] C. Zhao and S. Zhou, "Compact uniform attractors for dissipative lattice dynamical systems with delays," Discrete and Continuous Dynamical Systems Series A, vol. 21, no. 2, pp. 643663, 2008.

[29] T. Caraballo and K. Lu, "Attractors for stochastic lattice dynamical systems with a multiplicative noise," Frontiers of Mathematics in China, vol. 3, no. 3, pp. 317-335, 2008.

[30] Y. Lv and J. H. Sun, "Dynamical behavior for stochastic lattice systems," Chaos, Solitons \& Fractals, vol. 27, no. 4, pp. 1080-1090, 2006.

[31] A. Y. Abdallah, "Asymptotic behavior of the Klein-GordonSchrödinger lattice dynamical systems," Communications on Pure and Applied Analysis, vol. 5, no. 1, pp. 55-69, 2006.

[32] N. I. Karachalios and A. N. Yannacopoulos, "Global existence and compact attractors for the discrete nonlinear Schrödinger equation," Journal of Differential Equations, vol. 217, no. 1, pp. 88-123, 2005.
[33] C. Zhao and S. Zhou, "Compact kernel sections for nonautonomous Klein-Gordon-Schrödinger equations on infinite lattices," Journal of Mathematical Analysis and Applications, vol. 332, no. 1, pp. 32-56, 2007.

[34] X. Jia, C. Zhao, and X. Yang, "Global attractor and Kolmogorov entropy of three component reversible Gray-Scott model on infinite lattices," Applied Mathematics and Computation, vol. 218, no. 19, pp. 9781-9789, 2012.

[35] X. Yang, C. Zhao, and J. Cao, "Dynamics of the discrete coupled nonlinear Schrödinger-Boussinesq equations," Applied Mathematics and Computation, vol. 219, no. 16, pp. 8508-8524, 2013.

[36] C. Zhao and S. Zhou, "Compact kernel sections of long-waveshort-wave resonance equations on infinite lattices," Nonlinear Analysis: Theory, Methods \& Applications, vol. 68, no. 3, pp. 652670, 2008.

[37] C. Zhao and S. Zhou, "Limit behavior of global attractors for the complex Ginzburg-Landau equation on infinite lattices," Applied Mathematics Letters, vol. 21, no. 6, pp. 628-635, 2008.

[38] A. Mielke, "Bounds for the solutions of the complex GinzburgLandau equation in terms of the dispersion parameters," Physica D, vol. 117, no. 1-4, pp. 106-116, 1998.

[39] B. Wang, "The limit behavior of solutions for the Cauchy problem of the complex Ginzburg-Landau equation," Communications on Pure and Applied Mathematics, vol. 55, no. 4, pp. 481-508, 2002. 


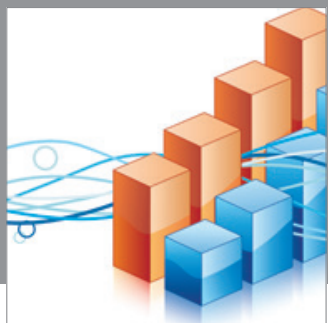

Advances in

Operations Research

mansans

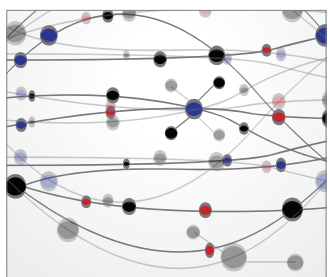

The Scientific World Journal
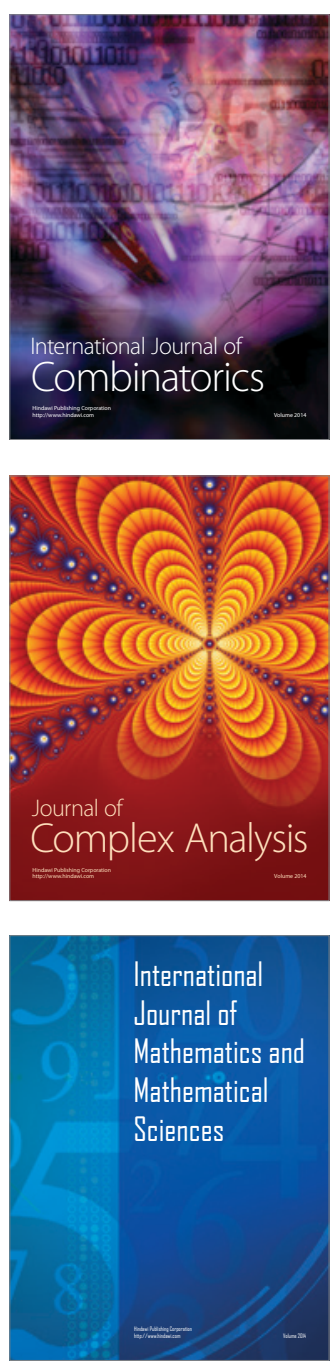
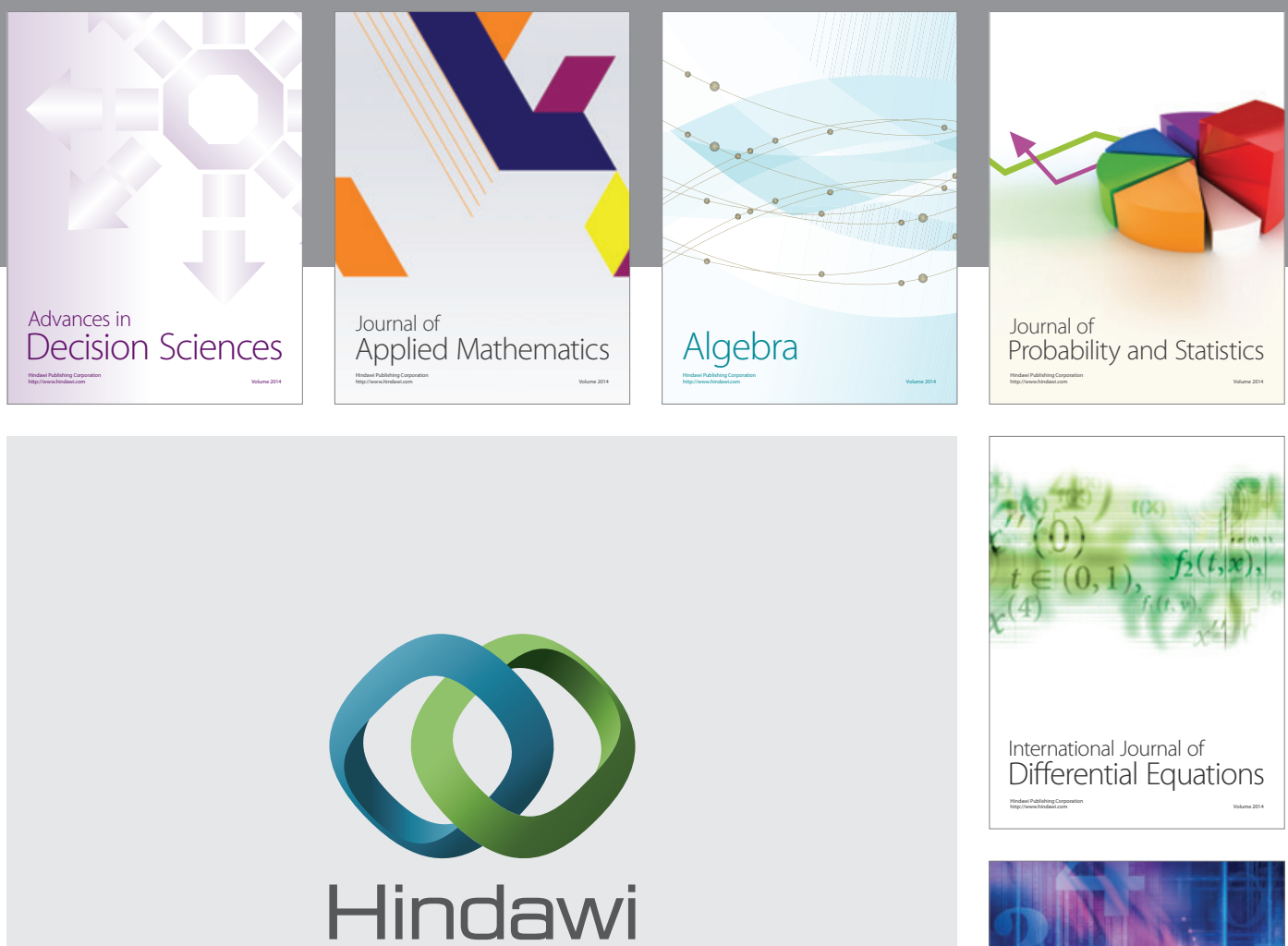

Submit your manuscripts at http://www.hindawi.com
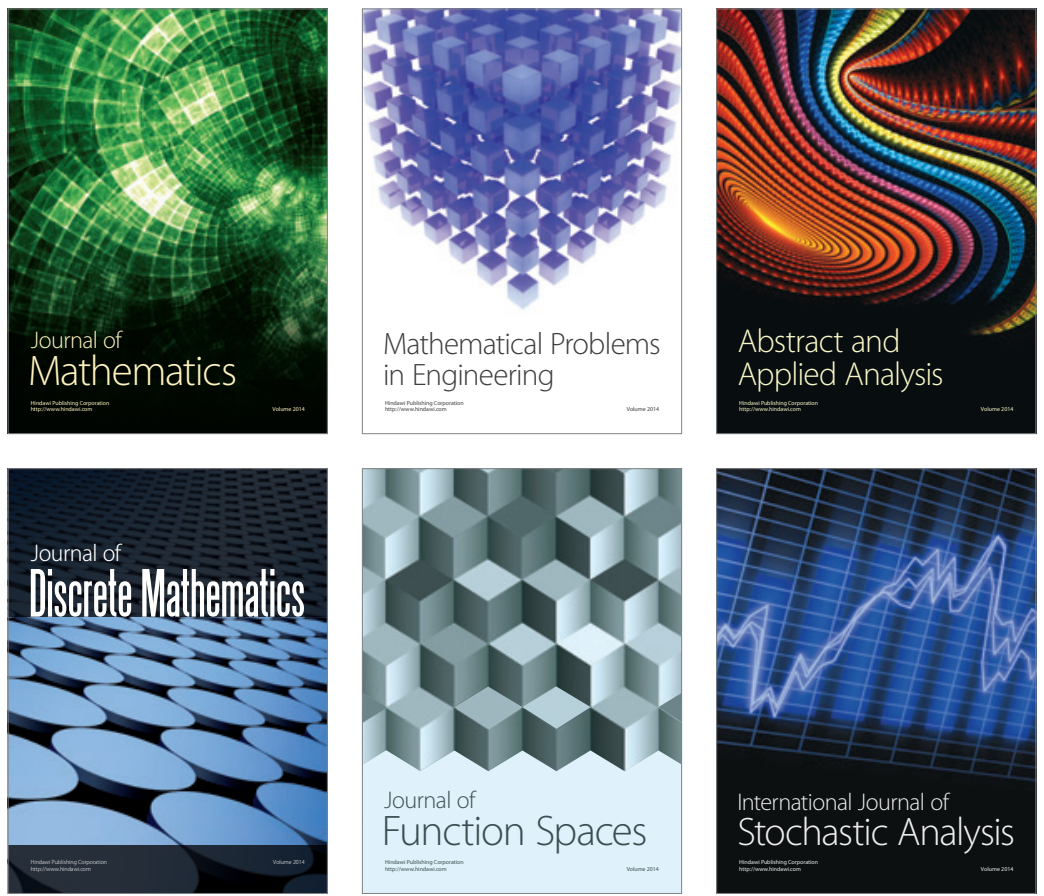

Journal of

Function Spaces

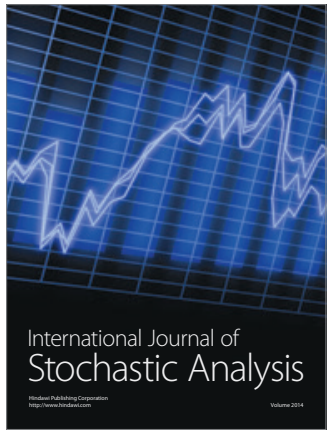

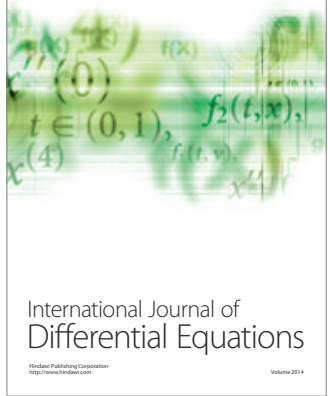
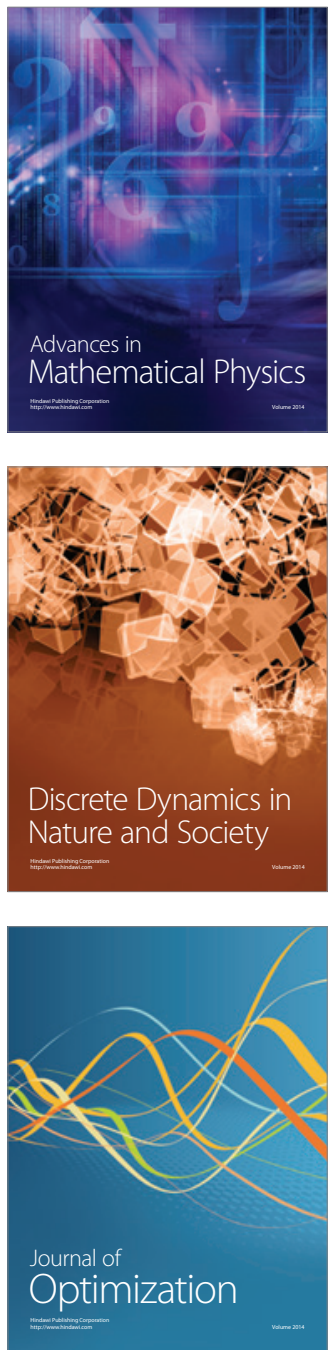